Original article

Section: Nutritional Research

\title{
Concentrations of Blood Serum and Urinal Ellagitannin Metabolites Depend Largely on the Post-Intake Time and Duration of Strawberry Phenolics Ingestion in Rats
}

\author{
Ewa Żary-Sikorska ${ }^{1 *}$, Monika Kosmala ${ }^{2}$, Joanna Milala², Bartosz Fotschki³ ${ }^{3}$ Katarzyna Ognik4 ${ }^{4}$ Jerzy Juśkiewicz $^{3}$ \\ ${ }^{1}$ Department of Microbiology and Food Technology, Faculty of Agriculture and Biotechnology University \\ of Science and Technology, Kaliskiego 7, 85-796 Bydgoszcz, Poland \\ ${ }^{2}$ Institute of Food Technology and Analysis, Łódź. University of Technology, Stefanowskiego 4/10, 90-924 Łódź, Poland \\ ${ }^{3}$ Department of Biological Functions of Food, Institute of Animal Reproduction and Food Research \\ of the Polish Academy of Sciences, Tuwima 10, 10-748 Olsztyn, Poland \\ ${ }^{4}$ Department of Biochemistry and Toxicology, Faculty of Biology, Animal Sciences and Bioeconomy, \\ University of Life Sciences, Akademicka 13, 20-950 Lublin, Poland
}

Key words: strawberry, ellagitannins, metabolites, urine, serum, rat

The different duration of a strawberry phenolic fraction intake and different post-intake time were experimental factors affecting the concentrations of ellagitannin metabolites in the urine and blood serum of rats. For four days, the animals were gavaged once a day as follows: group C (water, days 1-4), group F1-4 (fraction, days 1-4), group F1-3 (fraction, days 1-3; water, day 4), group F1-2 (fraction, days 1, 2; water, days 3, 4), group F3-4 (water, days 1, 2; fraction, days 3,4), and group F4 (water, days 1-3; and fraction, day 4). The daily dosage of the fraction gavaged to one rat was $20 \mathrm{mg} / \mathrm{kg}$ of body weight. The fraction contained monomers and dimers of ellagitannins as well as proanthocyanidins. The caecal, urinal, and blood serum ellagitannin metabolites were analysed $12 \mathrm{~h}$ after the last treatment. Ellagic acid, urolithin A, and nasutin A were detected in the caecal digesta. In turn, urolithin A, nasutin A, and their glucuronide conjugates were detected in the urine, while urolithin A glucuronide, nasutin A glucuronide, and ellagic acid dimethyl ether glucuronide were found in the serum. The highest caecal and urinal concentrations of ellagitannin metabolites followed the F1-4 treatment. In the serum, the highest concentrations of the metabolites were determined in the rats administered the phenolic fraction during days 1-4 and 3-4. No metabolites were found in the rats following the $C$ and F1-2 treatments. The results suggest that the presence of ellagitannin metabolites in the rat's urine and serum largely reflects the concentrations of caecal metabolites and the number of subsequent days of strawberry phenolic fraction administration.

\section{INTRODUCTION}

Worldwide, ellagitannins (ET) along with ellagic acid (EA) are ingested every day by consumers of fruit, seeds, and nuts. It is well known that pomegranates, berries, walnuts, almonds, grapes, sea buckthorn, and plenty of other plant sources contain high amounts of ET, being the hydrolysable class of tannins [Landete, 2011]. Ellagic acid, ET, and their derived metabolites are claimed to evoke a range of numerous beneficial physiological effects and are mostly referred as antioxidant, anti-inflammatory, and anti-pathogenic molecules that can act both locally in the gastrointestinal tract and systemically in the peripheral tissues of the host [Tomás-Barberán et al., 2017; Van de Velde et al., 2019]. From the chemical perspective, the ellagitannins are hexahydroxydiphenoyl esters of carbohydrates and cyclitols that may be subject to oligomerisation transformations [Milala et al., 2017]. The metabolic fate of ET in humans remains quite unknown although some ani-

\footnotetext{
* Corresponding Author:

E-mail: ezary@utp.edu.pl (E. Żary-Sikorska)
}

mal experiments have partly revealed those processes. It has been shown that part of plant ET may undergo hydrolysis to EA already in the stomach [Milala et al., 2017], while other researchers claimed ET to remain stable under conditions of that gastrointestinal segment [Sandhu et al., 2018]. There is no doubt that the large intestinal microbiota population with its huge enzymatic power acts as a paramount agent facilitating the hydrolysis of ET [Cerdá et al., 2005a,b; Ito et al., 2008; Sandhu et al., 2018]. Several researchers have reported that ET are not absorbed and prior to absorption must be metabolised by intestinal microbiota first into EA and then into other molecules such as urolithins and nasutin [Milala et al., 2017; Saha et al., 2016]. Urolithins contain a 6H-dibenzo[b,d]pyran-6-one nucleus with different phenolic hydroxyl groups. There are several types of urolithins, namely urolithins D, C, A, B with a decreasing number of the phenolic hydroxyl groups, respectively [Toney et al., 2019]. Following intestinal absorption and hepatic biotransformation, the main metabolites circulating in blood stream are urolithin A glucuronide and urolithin A aglycone [Kawabata et al., 2019]. The production of urolithins is a common result of consuming ET with 
a diet in almost all mammals, but some species (beavers, pigs, rats) are able to produce dehydroxyellagic acid derivatives, namely nasutins [González-Barrio et al., 2011].

Most in vivo experiments have shown data with regard to a continuous prolonged ingestion of berry ET [Larrosa et al., 2010; Giampieri et al., 2012]. Some authors reported that urolithins reach the maximum levels in human blood between $24 \mathrm{~h}$ and $48 \mathrm{~h}$ after ingestion of a meal containing ET, and their metabolites might be present in the blood serum and/or urine for the next $24 \mathrm{~h}$ [Larrosa et al., 2006] or even longer [Garcia-Munoz et al., 2014]. To our best knowledge, there are no results regarding the urinal and blood ET metabolites presence after different duration of polyphenols administration taking into account varied post-intake time points as well. Those knowledge gaps justify the need for a better recognition of the physiological and metabolic consequences of dietary ET administration. It should be noted that the chemical structure of ET and subsequently that of ET metabolites largely depends on the plant source [Landete, 2011; Karlińska et al., 2019]. The main strawberry ET is agrimoniin, a GOG-type dimer composed of two $\alpha$-1-O-galloyl-2,3:4,6-bis-hexahydroxydiphenoyl-D-glucose units linked by a C-O$\mathrm{C}$ bond between two gallic acid residues [Fotschki et al., 2018; Chiabrando et al., 2018; Nowicka et al., 2019]. The presence of sanguiin $\mathrm{H}-6$ and lambertianin $\mathrm{C}$, but as minor ET, has been also detected in strawberry fruit [Vrhovsek et al., 2012]. In the present study, it was hypothesised that the presence of ellagitannin metabolites in the blood serum and urine is strongly determined by their large intestinal metabolism following the post-intake time and the duration of strawberry phenolic fraction administration, and that these effects may be rapidly reversible after phenolic fraction withdrawal from a diet.

\section{MATERIAL AND METHODS}

\section{Preparation of strawberry phenolic fraction and its analysis}

The polyphenol-rich fraction was obtained from strawberry pomace, a common by-product obtained during juice production and provided by ALPEX Co. (Łęczeszyce, Poland). The details of strawberry phenolic fraction preparation were presented in the previous paper by Juśkiewicz et al. [2015]. In brief, the strawberry pomace was subjected to industrial vacuum drying at $70 \pm 2^{\circ} \mathrm{C}$ and then a seedless fraction was obtained with the aid of an appropriate set of sieves. That fraction was treated by a two-stage extraction with a $60 \%$ (v/v) aqueous solution of acetone. Next, after partial removal of the solvent via distillation, the resultant solutions were transferred onto a column packed with polymeric resin (Amberlite XAD 16, Sigma-Aldrich, Poznań, Poland). The water was used to elute the sugars and other water-soluble compounds from the extract loaded onto the column. The ellagitannin-rich fraction (F) was desorbed with a $40 \%$ aqueous solution of ethanol, concentrated to $c a$. $15 \%$ of dry matter, and then lyophilized. The detailed composition of the fraction was analysed based on the methods described below.

The official AOAC methods [Horwitz \& Latimer, 2007] were used to determine contents of: dry matter and ash
(940.26), protein (920.152), fat (930.09), and total dietary fibre (985.29). The concentration of ET, ellagic acid, anthocyanins, and flavonols were determined in the fraction after its dilution in methanol $(1 \mathrm{mg} / \mathrm{mL})$ using an HPLC system (Knauer Smartline system with photodiode array detector, Berlin, Germany) coupled with a Gemini C18 column (110 A, $250 \times 4.60 \mathrm{~mm} ; 5 \mu \mathrm{m}$, Phenomenex, Torrance, CA, USA). Phase A was $0.05 \%(\mathrm{v} / \mathrm{v})$ phosphoric acid in water, phase B was $0.05 \%(\mathrm{v} / \mathrm{v})$ phosphoric acid in $80 \%(\mathrm{v} / \mathrm{v})$ acetonitrile, the flow rate was $1.25 \mathrm{~mL} / \mathrm{min}$, the sample volume was $20 \mu \mathrm{L}$, and the temperature was $35^{\circ} \mathrm{C}$. Gradient: stabilization for 5 min with $4 \%$ of phase B, $4-15 \%$ B for $5-12.5$ min, $15-40 \%$ B for $12.5-42.5 \mathrm{~min}, 40-50 \% \mathrm{~B}$ for $42.5-51.8 \mathrm{~min}, 50-55 \% \mathrm{~B}$ for 51.8-53.4 $\mathrm{min}$ and 4\% B for 53.4-55 min. The following standards were used for phenolics identification: ellagic acid, flavonols (quercetin-3-O--glucoside, kaempferol-3-O-glucoside, quercetin, kaempferol, tiliroside), pelargonidin-3-O-glucoside (all from Extrasynthese, Genay, France), $p$-coumaric acid (Sigma-Aldrich), and ETs (hexahydroxydiphenoyl-D-glucose and agrimoniin) obtained by semi-preparative HPLC as described by Sójka et al. [2013]. The absorbance was measured at $280 \mathrm{~nm}$ (p-coumaric acid, tiliroside, hexahydroxydiphenoyl-D-glucose and agrimoniin), $360 \mathrm{~nm}$ (ellagic acid, quercetin, kaempferol and kaempferol glycosides), and $520 \mathrm{~nm}$ (anthocyanins).

The concentration of proanthocyanidins in the fraction was determined with the HPLC method after their hydrolysis in an acidic environment with an excess of phloroglucinol, according to Kennedy \& Jones [2001]. The obtained reaction products were analysed using a Knauer Smartline chromatograph (Berlin, Germany) equipped with a UV-Vis detector (PDA 280, Knauer, Berlin, Germany) and a fluorescence detector (Shimadzu RF-10Axl, Kyoto, Japan), and coupled with a Gemini C18 column (110, $250 \times 4.60 \mathrm{~mm} ; 5 \mu \mathrm{m}$, Phenomenex, Torrance, CA, USA). The separation conditions were as earlier described by Kosmala et al. [2015]. The identification was performed at $280 \mathrm{~nm}$ using a UV-Vis detector and the following standards: (-)-epicatechin, (+)-catechin, (-)-epigallocatechin and their respective phloroglucinol adducts. Quantification was conducted based on peak areas recorded using a fluorescence detector (excitation wavelength: $278 \mathrm{~nm}$; emission wavelength: $360 \mathrm{~nm}$ ). Standard curves of (-)-epicatechin and (+)-catechin for terminal units and (-)-epicatechin-phloroglucinol adduct for extender units were used to quantify the proanthocyanidin breakdown products.

\section{The gavage in vivo experiment}

The in vivo experiment was conducted on 48 adult male Wistar outbred rats (Rattus norvegicus, Cmdb:WI). The animals were used in compliance with the European Guidelines for the Care and Use of Laboratory Animals [Directive $63 \mathrm{EU}$, 2010]. The experimental protocol was approved by the Local Animal Care and Use Committee (Approval No. 10/2018; Olsztyn, Poland). All efforts were made to minimise the suffering of experimental animals. Two weeks before and during the gavage experiment, the rats were fed with a polyphenol-free diet prepared in a laboratory scale based on recommendation of AIN-1993 [Reeves, 1997]. This diet contained about 14.8\% casein (Lacpol Co., Murowana Goślina, Poland), 
TABLE 1. Experimental treatments ${ }^{\#}$ with the strawberry phenolic fraction gavaged to rats for 4 days.

\begin{tabular}{l|c|c|c|c|c}
\hline Group & $\begin{array}{c}\text { Day 1; } \\
8 \text { p.m. }\end{array}$ & $\begin{array}{c}\text { Day 2; } \\
8 \text { p.m. }\end{array}$ & $\begin{array}{c}\text { Day 3; } \\
8 \text { p.m. }\end{array}$ & $\begin{array}{c}\text { Day 4; } \\
8 \text { p.m. }\end{array}$ & $\begin{array}{c}\text { Day 5; } \\
8 \text { a.m. }\end{array}$ \\
\hline C & Water & Water & Water & Water & $*$ \\
F1-4 & Fraction & Fraction & Fraction & Fraction & $*$ \\
F1-3 & Fraction & Fraction & Fraction & Water & $*$ \\
F1-2 & Fraction & Fraction & Water & Water & $*$ \\
F3-4 & Water & Water & Fraction & Fraction & $*$ \\
F4 & Water & Water & Water & Fraction & $*$ \\
\hline
\end{tabular}

*Urine, blood, and caecal digesta collection (at the termination of the study). ${ }^{\text {TT }}$ The dosage of the strawberry phenolic fraction gavaged to a rat was $20 \mathrm{mg} / \mathrm{kg}$ of $\mathrm{BW}$.

0.2\% DL-methionine (SIGMA, Poznań, Poland), 8\% rapeseed oil, $6 \%$ cellulose, standard mineral and vitamin mixes ( 3.5 and $1 \%$, respectively; according to AIN-93 requirements of laboratory adult rats), $0.2 \%$ choline chloride, and corn starch up to $100 \%$. The energy contribution of main dietary components was as follows: $16.1 \%$ by protein, 63.9 by $\mathrm{N}$-free extractives, and $20 \%$ by fat.

The rats were similar in the body weight (BW) values $(245 \pm 12.6 \mathrm{~g})$. The gavage experiment lasted 5 days (see experimental scheme presented in Table 1). A fresh diet was served every day ad libitum and access to water was continuous. Standard conditions at a temperature of $21-22^{\circ} \mathrm{C}$ and relative air humidity of $50 \%$ with intensive room ventilation $(15 \times$ per $h)$, and a $12 \mathrm{~h}$ lighting/12 h dark regimen were applied. During the period of 1-4 days, every day at 8 p.m., the rats were subjected to the following treatments $(n=8)$ without or with the strawberry ellagitannin-rich fraction gavaged intragastrically: group C (control with water all four days), group F1-4 (fraction administered all four days), group F1-3 (fraction gavaged days 1, 2, 3, and water gavaged day 4), group F1-2 (fraction gavaged days 1, 2, and water gavaged days 3, 4), group F3-4 (water gavaged days 1, 2, and fraction gavaged days 3, 4), and group F4 (water gavaged days 1, 2, 3, and fraction gavaged day 4). The dosage of the strawberry phenolic fraction gavaged to a rat was $20 \mathrm{mg} / \mathrm{kg}$ of BW and it reflected an ingestion of a half kilogram of fresh strawberry with average phenolics concentration by an adult weighing $65 \mathrm{~kg}$ (calculated with the aid of Body Surface Area method; Wang \& Hihara, [2004]). The amount of water or a phenolic fraction solution gavaged to a rat was prepared in a way that an animal weighing $250 \mathrm{~g}$ was treated with $1 \mathrm{~mL}$. The animals were treated according to recommendations for refinements for intragastric gavage in rats [Okva et al., 2006].

At the termination of the study, on the fifth day 8 a.m., the rats were anaesthetised with a ketamine/xylazine solution in $0.9 \% \mathrm{NaCl}$ (100 and $10 \mathrm{mg} / \mathrm{kg} \mathrm{BW}$, respectively). After laparotomy, blood samples were taken from the caudal vena cava. The caecum was removed and fresh digesta was frozen in liquid nitrogen, then stored at $-80^{\circ} \mathrm{C}$ until analyses. The serum was obtained by blood centrifugation (MPW-352R; MPW MED. INSTRUMENTS, Warsaw Poland) for $15 \mathrm{~min}$ at $380 \times g$. The urine was collected directly from the urinal bladder, then serum and urinal samples were stored under the same conditions as caecal digesta. In the digesta samples, free ellagic acid and ET metabolites were extracted twice with the aid of $100 \%$ and $70 \%$ (v/v) acetone. The details of extraction and HPLC analyses of caecal metabolites were as earlier described by Milala et al. [2017]. Urine samples (1-2 mL) were acidified with $0.125 \mathrm{~mL}$ of $1 \mathrm{~mol} / \mathrm{L} \mathrm{H}_{3} \mathrm{PO}_{4}$ and injected to a $60 \mathrm{mg}$ SPE Strata X-33 column conditioned according to the manufacturer's instructions (Phenomenex, Torrance, CA). The column was washed with $3 \mathrm{~mL}$ of water, $3 \mathrm{~mL}$ of $0.1 \mathrm{~mol} / \mathrm{L}$ acetate buffer, and $6 \mathrm{~mL}$ of water, and dried. The analyte was eluted with methanol $(2 \times 0.5 \mathrm{~mL})$ and analysed by UHPLC-MS using a Dionex UltiMate 3000 UHPLC and a Thermo Scientific Q Exactive series quadrupole ion trap mass spectrometer. The column was a Kinetex C18 (100 A; $150 \times 2.1 \mathrm{~mm} ; 2.6 \mu \mathrm{m}$, Phenomenex, Torrance, CA, USA) kept at $35^{\circ} \mathrm{C}$. A binary gradient of $0.1 \%(\mathrm{v} / \mathrm{v})$ formic acid in water as a mobile phase A and $0.1 \%(\mathrm{v} / \mathrm{v})$ formic acid in acetonitrile as a mobile phase $B$ was used at a flow rate of $0.5 \mathrm{~mL} / \mathrm{min}$. The gradient program was: $5 \%$ of mobile phase B for the first $1.44 \mathrm{~min}, 5-15 \%$ B from 1.44 to $2.98 \mathrm{~min}, 15-40 \%$ B from 2.98 to $10.1 \mathrm{~min}, 40-73 \% \mathrm{~B}$ from 10.1 to $11.5 \mathrm{~min}$, isocratic conditions at $73 \% \mathrm{~B}$ from 11.55 to $12.7 \mathrm{~min}, 73-5 \% \mathrm{~B}$ from 12.7 to $13.28 \mathrm{~min}$, and column equilibration at $5 \% \mathrm{~B}$ from 13.28 to $18 \mathrm{~min}$. Mass spectrometry analysis was performed in a negative ion mode under the following conditions: capillary voltage, $+4 \mathrm{kV}$; sheath gas pressure, 60 au (arbitrary units); auxiliary gas, $10 \mathrm{au}$; and scan range from 120 to $1200 \mathrm{~m} / \mathrm{z}$. Blood serum samples: $1 \mathrm{~mL}$ of acetone was added to $0.5 \mathrm{~mL}$ of serum; the mixture was mixed, sonicated for $10 \mathrm{~min}$, and centrifuged at $900 \mathrm{rad} / \mathrm{s}$ (ScanSpeed 40, LaboGene, Allerød, Denmark). The procedure was repeated with $1.0 \mathrm{~mL}$ of acetone, and the extracts were combined and evaporated to dryness under vacuum in a ScanSpeed 40 low speed centrifuge (LaboGene, Allerød, Denmark), lyophilised in an Alpha 1-2 LD plus freeze-dryer (Martin Christ Gefriertrocknungsanlagen GmbH, Osterode am Harz, Germany), solubilised in $0.2 \mathrm{~mL}$ of methanol, and then analysed by UHPLC-MS (parameters as in the analysis of urine). Detection of ellagitannin metabolites was performed at $360 \mathrm{~nm}$. Standards used were ellagic acid, urolithin A, and urolithin B (Sigma-Aldrich, Steinheim, Germany). Urolithin A glucuronide was prepared as described by Cerdá et al. [ 2005a]. The raw material for the isolation of urolithin A glucuronide was two litres of urine from three healthy volunteers $(25-45$ years old) who consumed $400 \mathrm{~g}$ of strawberry per day, as described in Fotschki et al. [2014]. Nasutin was isolated from the faeces of rats fed ellagitannin preparation in the same manner. Identification of metabolites was confirmed by HPLC-MS and compared with literature findings [González-Barrio et al., 2011; GarcíaVillalba et al., 2016; Jurgoński et al., 2017; Milala et al., 2017].

\section{Statistical analysis}

The results are expressed as the means \pm SEM except for the chemical composition of the strawberry phenolic fraction, which is expressed as the means \pm SD. The Kruskal-Wallis one-way ANOVA by ranks was used followed by Dunn's post hoc test $(P \leq 0.05)$. The statistical analysis was performed 
using STATISTICA software, version 12.0 (StatSoft Corp., Kraków, Poland).

\section{RESULTS AND DISCUSSION}

Ellagitannins are abundant in many berries, including strawberries, and after consumption those polyphenolic compounds are degraded to their monomers throughout the gastrointestinal segments, and then metabolised by gut microbiota to $6 H$-dibenzo $[b, d]$ pyran-6-one derivatives, i.e., urolithins, nasutins, depending on the host species [Fotschki et al., 2015; Kosmala et al., 2017; Milala et al., 2017]. The ET metabolites could be further conjugated to glucuronides and sulfates in the intestine and liver before entering the systemic circulation [Sandhu et al., 2018]. Some authors reported that large intestinal bacteria were able to directly metabolise the native ET molecules without their hydrolysis in the upper gastrointestinal tract [Piwowarski et al., 2016]. The recent experiment conducted by Milala et al. [2017] demonstrated that the metabolism of ellagitannins with different degrees of polymerization may result in some changes in the urinal and blood serum metabolite profile. Despite those differences, the aforementioned and other authors clearly showed that after strawberry consumption the main metabolites in the rat blood serum are urolithin A glucuronide, nasutin A glucuronide, and ellagic acid dimethyl ether glucuronide (DMEAG) while the urinal metabolites are represented by urolithin A, nasutin A, urolithin A glucuronide, nasutin A glucuronide, and nasutin iso-glucuronide [Fotschki et al., 2016]. The analysis of the current knowledge allows to conclude that the resultant production of ET metabolites is difficult to predict accurately. But, if the ET composition of a certain natural product is known, the formation of gut microbiota metabolites might be quite precisely assessed [Piwowarski et al., 2016]. In the present experiment, the strawberry phenolic fraction gavaged to rats contained monomeric and dimeric ellagitannins, and proanthocyanidins as the main polyphenolic constituents, and small amounts of ellagic acid and flavonols (Table 2).
TABLE 2. Chemical composition of the strawberry phenolic fraction gavaged to experimental rats.

\begin{tabular}{lc}
\hline Components & Content $(\mathrm{g} / 100 \mathrm{~g})$ \\
\hline Dry matter & $91.31 \pm 0.05$ \\
Ash & $0.03 \pm 0.04$ \\
Fat & $0.00 \pm 0.00$ \\
Protein & $1.83 \pm 0.03$ \\
Other components* & $7.17 \pm 0.01$ \\
Total polyphenols & $82.3 \pm 0.10$ \\
Ellagic acid & $0.20 \pm 0.00$ \\
ETs & $57.3 \pm 0.10$ \\
\multicolumn{1}{c}{ Monomers } & $23.3 \pm 0.10$ \\
Dimers & $34.0 \pm 0.10$ \\
Proanthocyanidins & $23.9 \pm 0.20$ \\
Anthocyanins & $0.00 \pm 0.00$ \\
Flavonols & $0.90 \pm 0.00$ \\
\hline
\end{tabular}

The results are expressed as the means $\pm S D, n=3$. ETs, ellagitannins *Low-molecular carbohydrates and structural components of plant cell walls, including dietary fibre.

The formation of urolithin A and nasutin A in the caecal digesta followed the experimental gavage treatments (Table 3). In the digesta, ellagic acid was also present, and it could have originated both directly from the phenolic fraction and from the bacterial hydrolysis of ET. The dominant caecal metabolite was nasutin A followed by urolithin A. Such results were in agreement with other studies conducted on laboratory rats fed diets containing strawberry products or extracts [Fotschki et al., 2014, 2016; Jurgoński et al., 2017; Kosmala et al., 2015; Milala et al., 2017; TomásBarberán et al., 2017]. The caecal concentration of total ET metabolites was the highest in the $\mathrm{F} 1-4$ rats $(P<0.05$ vs.

TABLE 3. Caecal EA and ET metabolites presence in rats differently gavaged the strawberry phenolic fraction for four days ( $\mu \mathrm{g} / \mathrm{g}$ digesta).

\begin{tabular}{|c|c|c|c|c|}
\hline Group* & Ellagic acid & Urolithin A & Nasutin A & Sum of metabolites \\
\hline $\mathrm{C}$ & $0.00 \pm 0.00^{c}$ & $0.00 \pm 0.00^{\mathrm{d}}$ & $0.00 \pm 0.00^{\mathrm{d}}$ & $0.00 \pm 0.00^{\mathrm{d}}$ \\
\hline F1-4 & $0.856 \pm 0.089^{a}$ & $45.1 \pm 7.22^{\mathrm{a}}$ & $122 \pm 18.5^{\mathrm{a}}$ & $168 \pm 17.2^{\mathrm{a}}$ \\
\hline F1-3 & $0.422 \pm 0.066^{\mathrm{b}}$ & $18.2 \pm 11.1^{\mathrm{c}}$ & $25.6 \pm 5.12^{c}$ & $44.2 \pm 10.4^{c}$ \\
\hline F1-2 & $0.056 \pm 0.043^{c}$ & $1.02 \pm 0.891^{\mathrm{d}}$ & $1.25 \pm 0.880^{\mathrm{d}}$ & $2.33 \pm 0.885^{\mathrm{d}}$ \\
\hline F3-4 & $0.815 \pm 0.095^{\mathrm{a}}$ & $39.2 \pm 7.89^{\mathrm{ab}}$ & $96.1 \pm 11.8^{\mathrm{ab}}$ & $136.1 \pm 10.4^{\mathrm{ab}}$ \\
\hline $\mathrm{F} 4$ & $0.803 \pm 0.102^{\mathrm{a}}$ & $31.2 \pm 10.9^{\mathrm{b}}$ & $85.9 \pm 10.4^{b}$ & $117.9 \pm 9.22^{b}$ \\
\hline $\mathrm{P}$ value & $<0.001$ & $<0.001$ & $<0.001$ & $<0.001$ \\
\hline
\end{tabular}

*The rats were subjected to the following treatments $(n=8)$ without or with the strawberry phenolic fraction (see Table 1) gavaged intragastrically: group C (control with water all four days), group F1-4 (fraction administered all four days), group F1-3 (fraction gavaged days 1, 2, 3, and water gavaged day 4), group F1-2 (fraction gavaged days 1, 2, and water gavaged days 3, 4), group F3-4 (water gavaged days 1, 2, and fraction gavaged days 3,4 ), and group F4 (water gavaged days $1,2,3$, and fraction gavaged day 4). The caecal samples were collected on the $5^{\text {th }}$ day $12 \mathrm{~h}$ after the last gavage treatment. Values (presented as mean $\pm \mathrm{SEM}$ ) within each column with the same superscript letter are not different at $\mathrm{P}<0.05$. EA, ellagic acid; ET, ellagitannins. 
TABLE 4. Urinal EA and ET metabolites presence in rats differently gavaged the strawberry phenolic fraction for four days $(\mu \mathrm{g} / \mathrm{L})$.

\begin{tabular}{lcccccc}
\hline Group* & Urolithin A & Nasutin A & Urolithin A glucuronide & Nasutin A glucuronide & Sum of metabolites \\
\hline C & $0.00 \pm 0.00^{\mathrm{c}}$ & $0.00 \pm 0.00^{\mathrm{d}}$ & $0.00 \pm 0.00^{\mathrm{d}}$ & $0.00 \pm 0.00^{\mathrm{d}}$ & $0.00 \pm 0.00^{\mathrm{d}}$ \\
F1-4 & $111 \pm 56.0^{\mathrm{a}}$ & $70.1 \pm 12.4^{\mathrm{a}}$ & $889 \pm 25.9^{\mathrm{a}}$ & $569 \pm 12.2^{\mathrm{a}}$ & $1639 \pm 234^{\mathrm{a}}$ \\
F1-3 & $22.0 \pm 12.4^{\mathrm{b}}$ & $18.2 \pm 11.1^{\mathrm{c}}$ & $77.4 \pm 29.0^{\mathrm{c}}$ & $88.9 \pm 32.1^{\mathrm{c}}$ & $206 \pm 27.4^{\mathrm{c}}$ \\
F1-2 & $0.00 \pm 0.00^{\mathrm{c}}$ & $0.00 \pm 0.00^{\mathrm{d}}$ & $0.00 \pm 0.00^{\mathrm{d}}$ & $0.00 \pm 0.00^{\mathrm{d}}$ & $0.00 \pm 0.00^{\mathrm{d}}$ \\
F3-4 & $102 \pm 45.8^{\mathrm{a}}$ & $69.2 \pm 21.8^{\mathrm{a}}$ & $758 \pm 288^{\mathrm{ab}}$ & $469 \pm 174^{\mathrm{ab}}$ & $1398 \pm 233^{\mathrm{ab}}$ \\
F4 & $95.1 \pm 27.7^{\mathrm{a}}$ & $44.2 \pm 20.9^{\mathrm{b}}$ & $655 \pm 201^{\mathrm{b}}$ & $409 \pm 137^{\mathrm{b}}$ & $1203 \pm 174^{\mathrm{b}}$ \\
\hline P value & 0.009 & $<0.001$ & $<0.001$ & $<0.001$ & $<0.001$ \\
\hline
\end{tabular}

*The rats were subjected to the following treatments $(n=8)$ without or with the strawberry phenolic fraction (see Table 1) gavaged intragastrically: group C (control with water all four days), group F1-4 (fraction administered all four days), group F1-3 (fraction gavaged days 1, 2, 3, and water gavaged day 4), group F1-2 (fraction gavaged days 1, 2, and water gavaged days 3, 4), group F3-4 (water gavaged days 1, 2, and fraction gavaged days 3,4 ), and group F4 (water gavaged days 1,2 , 3, and fraction gavaged day 4). The caecal samples were collected on the $5^{\text {th }}$ day $12 \mathrm{~h}$ after the last gavage treatment. Values (presented as mean \pm SEM) within each column with the same superscript letter are not different at $P<0.05$. EA, ellagic acid; ET, ellagitannins.

other groups except F3-4 rats; Table 3). In regard to the respective caecal ET metabolites, the observed significance of differences in nasutin A and urolithin A concentrations between the treatments was similar to that noted for the sum of all caecal metabolites. The highest concentration of EA in the caecal digesta was in the F1-4, F3-4, and F4 groups ( $P<0.05$ vs. remaining groups). Additionally, the F1-3 rats had significantly higher caecal EA concentration than those from the $\mathrm{C}$ and $\mathrm{F} 1-2$ groups.

Nasutin A has been hardly often detected in intestinal digesta and faeces of humans, and urolithin A and urolithin B are predominant ET bacterial metabolites [González-Barrio et al., 2011]. Certainly, some variations among ET consumers have been observed, and those differences are ascribed to the different profile of the intestinal bacteria population [Cerda et al., 2005a; Romo-Vaquero et al., 2019]. In the intestine of several species producing nasutins, e.g. termites, beavers, rats, pigs, free or released EA is undergoing microbial dihydroxylation but without lactone ring opening leading to nasutins formation, i.e. metabolites with two lactone rings (as in EA) but without two hydroxyl groups [Espín et al., 2013]. The products of bacterial ET metabolism absorbed in the upper and lower gastrointestinal tract, then glucuronidated in the portal circulation, eventually turn up in the urine [Milala et al., 2017]. In the present experiment, urolithin A, nasutin A, urolithin A glucuronide, nasutin A glucuronide were detected in the urine of rats treated with the strawberry phenolic fraction, while urolithin A glucuronide, nasutin A glucuronide and DMEAG were found in their blood serum (Tables 4 and 5). It has been reported that in the case of rats the production of nasutin $\mathrm{A}$ and its glucuronide was elevated upon the consumption of bis-hexahydroxydiphenoyl-D-glucose (monomeric ET) and agrimoniin (dimeric ET), while dietary EA caused increased concentrations of urolithin A and its glucuronide in the gastrointestinal digesta as well as the inner biological fluids: blood, lymph, and urine [Jurgoński et al., 2017]. Our results showed that urolithin A and urolithin A glucuronide were present in the urine of rats despite the fact that the phenolic fraction contained only trace amounts of EA. Urolithin A was also the main metabolite detected in the blood serum. In the urine, the predominant metabolites were glucuronidated urolithin A and nasutin A. It has been reported that glucuronidation enhances the solubility of these molecules and facilitates their urinal excretion [Fotschki et al., 2018].

Many authors while conducting studies on human volunteers but also on laboratory rodents observed huge differences between individuals within a group in relation to concentrations of ET metabolites [Kosmala et al., 2017; Tomás-Barberán et al., 2017]. In the present experiment, the standard error of the mean (SEM) values calculated for each group, and each metabolite were not as high as expected, hence the differences observed between treatments were statistically significant, thus giving the opportunity to observe biological relationships in the ET metabolites formation.

The animals from the F1-4 group had the highest total ET metabolites concentration in the urine $(P<0.05$ vs. other groups except F3-4 rats; Table 4). Considering respective ET metabolites, the urinal urolithin A concentrations determined in groups F1-4, F3-4, and F4 significantly excelled those determined in the remaining groups $(P<0.05)$; additionally, the F1-4 group had a significantly higher concentration of total ET metabolites in the urine vs. F4 group. It should be noted that apart from control rats (group C) treated only with water, the animals from group F1-2 had no metabolites in their urine and blood serum as well. As for nasutin A in the urine, the F4 rats had a significantly lower concentration of that metabolite in comparison to the rats from groups F1-4 and F3-4, but significantly higher one compared to the rats following $\mathrm{C}, \mathrm{F} 1-2$, and $\mathrm{F} 1-3$ treatments $(P<0.05)$. The significance of differences in urinal urolithin A glucuronide and nasutin A glucuronide concentrations between the treatments was identical as it was for the sum of metabolites in the urine $\left(\mathrm{F} 1-4^{\mathrm{a}}>\mathrm{F} 3-4^{\mathrm{ab}}>\mathrm{F} 4^{\mathrm{b}}>\mathrm{F} 1-3^{\mathrm{c}}>\mathrm{F} 1-2^{\mathrm{d}}, \mathrm{C}^{\mathrm{d}}\right)$.

The blood serum analyses of ET metabolites revealed that the total concentration metabolites, as well as concentrations of urolithin A glucuronide and DMEAG were the highest 
TABLE 5. Blood serum EA and ET metabolites presence in rats differently gavaged the strawberry phenolic fraction for four days $(\mu \mathrm{g} / \mathrm{L})$.

\begin{tabular}{l|c|c|c|c}
\hline Group* & $\begin{array}{c}\text { Urolithin A } \\
\text { glucuronide }\end{array}$ & $\begin{array}{c}\text { Nasutin A } \\
\text { glucuronide }\end{array}$ & $\begin{array}{c}\text { Ellagic acid } \\
\text { dimethyl } \\
\text { ether } \\
\text { glucuronide } \\
\text { DMEAG }\end{array}$ & $\begin{array}{c}\text { Sum of } \\
\text { metabolites }\end{array}$ \\
\hline C & $0.00 \pm 0.00^{\mathrm{c}}$ & $0.00 \pm 0.00^{\mathrm{d}}$ & $0.00 \pm 0.00^{\mathrm{c}}$ & $0.00 \pm 0.00^{\mathrm{c}}$ \\
F1-4 & $44.4 \pm 15.9^{\mathrm{a}}$ & $14.0 \pm 8.22^{\mathrm{a}}$ & $9.11 \pm 2.99^{\mathrm{a}}$ & $67.1 \pm 18.8^{\mathrm{a}}$ \\
F1-3 & $7.32 \pm 2.22^{\mathrm{c}}$ & $1.01 \pm 0.00^{\mathrm{d}}$ & $0.00 \pm 0.00^{\mathrm{c}}$ & $8.08 \pm 2.09^{\mathrm{c}}$ \\
F1-2 & $0.00 \pm 0.00^{\mathrm{c}}$ & $0.00 \pm 0.00^{\mathrm{d}}$ & $0.00 \pm 0.00^{\mathrm{c}}$ & $0.00 \pm 0.00^{\mathrm{c}}$ \\
F3-4 & $41.4 \pm 25.9^{\mathrm{a}}$ & $9.04 \pm 1.00^{\mathrm{b}}$ & $8.05 \pm 3.12^{\mathrm{a}}$ & $58.3 \pm 26.5^{\mathrm{a}}$ \\
F4 & $25.1 \pm 8.19^{\mathrm{b}}$ & $4.04 \pm 1.02^{\mathrm{c}}$ & $5.13 \pm 1.22^{\mathrm{b}}$ & $34.4 \pm 9.49^{\mathrm{b}}$ \\
\hline P value & 0.004 & 0.041 & 0.024 & 0.021 \\
\hline
\end{tabular}

*The rats were subjected to the following treatments $(n=8)$ without or with the strawberry phenolic fraction (see Table 1) gavaged intragastrically: group C (control with water all four days), group F1-4 (fraction administered all four days), group F1-3 (fraction gavaged days 1, 2, 3, and water gavaged day 4), group F1-2 (fraction gavaged days 1, 2 , and water gavaged days 3,4 ), group F3-4 (water gavaged days 1,2 , and fraction gavaged days 3,4 ), and group F4 (water gavaged days 1, 2, 3, and fraction gavaged day 4). The caecal samples were collected on the $5^{\text {th }}$ day $12 \mathrm{~h}$ after the last gavage treatment. Values (presented as mean \pm SEM) within each column with the same superscript letter are not different at $P<0.05$. EA, ellagic acid; ET, ellagitannins.

in groups $\mathrm{F} 1-4$ and $\mathrm{F} 3-4$ ( $P<0.05$ vs. remaining treatments), followed by the $\mathrm{F} 4$ group $(P<0.05$ vs. all other groups; Table 5). In the case of blood serum, the highest concentration of nasutin A glucuronide was determined in the F1-4 rats ( $P<0.05$ vs. others), followed by F3-4 ( $P<0.05$ vs. others), and F4 ( $P<0.05$ vs. others) rats. The above-mentioned results support the accepted hypothesis that the presence of ellagitannin metabolites in the blood serum and urine is strongly determined by the post-intake time and the duration of strawberry phenolic fraction administration, as well as that these effects may be rapidly reversible after phenolic fraction withdrawal from a diet. The latter statement is justified especially by results obtained in the F1-2 treatment which was accompanied by no ET metabolites detected both in the urine and the blood serum. That statement was also supported by comparison of results achieved for rats from group F4 vs. F1-3, the former had significantly more ET metabolites despite ingesting the strawberry phenolic fraction only once but $12 \mathrm{~h}$ prior analyses, and the F1-3 rats were treated three times with the fraction but the last treatment before ET metabolites analysis in that group was with water. In that context, other experiments clearly showed that ellagitannin and ellagic acid metabolites are detected in blood and urine already one day after consumption of a diet containing strawberry polyphenols [Milala et al., 2017]. In the study reported by Ito [2011], the urinal excretion of metabolites continued up to $72 \mathrm{~h}$ after oral administration of geraniin but the dosage $(50 \mathrm{mg} / \mathrm{rat})$ was much more higher than it was in our study (20 mg of strawberry phenolic fraction per $\mathrm{kg}$ of $\mathrm{BW}$ ).

\section{CONCLUSIONS}

In conclusion, the performed experiment on rats gavaged once a day the strawberry phenolic fraction or water showed that the concentration of strawberry ellagitannin metabolites in the rat's biological fluids, i.e. urine and blood serum, reflected metabolites content in the caecal digesta and depended on the number of subsequent days of phenolic fraction administration. The presence of those metabolites in the caecal digesta, urine, and serum is quickly reversible after phenolic fraction withdrawal from a diet.

\section{CONFLICT OF INTEREST}

The authors declare no conflict of interest.

\section{REFERENCES}

1. Cerdá, B., Periago, P., Espín, J.C., Tomás-Barberan, F.A. (2005a). Identification of urolithin A as a metabolite produced by human colon microflora from ellagic acid and related compounds. Journal of Agricultural and Food Chemistry, 53(14), 5571-5576.

2. Cerdá, B., Tomás-Barberán, F.A., Espín, J.C. (2005b). Metabolism of antioxidant and chemopreventive ellagitannins from strawberries, raspberries, walnuts and oak-aged wine in humans: identification of biomarkers and individual variability. Journal of Agricultural and Food Chemistry, 53(2), 227-235.

3. Chiabrando, V., Giuggioli, N., Maghenzani, M., Peano, C., Giacalone, G. (2018). Improving storability of strawberries with gaseous chlorine dioxide in perforated clamshell packaging. Polish Journal of Food and Nutrition Sciences, 68(2), 141-148.

4. Directive 2010/63/EU of the European Parliament and of the Council of 22 September 2010 on the protection of animals used for scientific purposes (2010). Official Journal of the European Union, L 276, 20.10.2010, p. 33-79.

5. Espín, J.C., Larrosa, M., Garcia-Conesa, M.T., Tomas-Barberán, F.A. (2013). Biological significance of urolithins, the gut microbial ellagic acid-derived metabolites: the evidence so far. Evidence-Based Complementary and Alternative Medicine: eCAM, 2013, art. no. 270418.

6. Fotschki, B., Juśkiewicz, J., Jurgoński, A., Kołodziejczyk, K., Milala, J., Kosmala, M., Zduńczyk, Z. (2016). Anthocyanins in strawberry polyphenolic extract enhance the beneficial effects of diets with fructooligosaccharides in the rat cecal environment. PLoS ONE, 11 (2), art. no. e0149081.

7. Fotschki, B., Juśkiewicz, J., Kołodziejczyk K., Jurgoński A., Kosmala M., Milala, J., Ognik K., Zduńczyk, Z. (2018). Protective effects of ellagitannin-rich strawberry extracts on biochemical and metabolic disturbances in rats fed a diet high in fructose. Nutrients, 10(4), art. no. 445.

8. Fotschki, B., Juśkiewicz, J., Sójka, M., Jurgoński, A., Zduńczyk, Z. (2015). Ellagitannins and flavan-3-ols from raspberry pomace modulate caecal fermentation processes and plasma lipid parameters in rats. Molecules, 20(12), 22848-22862.

9. Fotschki, B., Milala, J., Karlińska, E., Zduńczyk, Z., Juśkiewicz, J. (2014). Strawberry ellagitannins thwarted the positive effects of dietary fructooligosaccharides in rat cecum. Journal of Agricultural and Food Chemistry, 62(25), (SI), 5871-5880. 
10. Garcia-Munoz, C., Hernandez, L., Perez, A., Vaillant, F. (2014). Diversity of urinary excretion patterns of main ellagitannins' colonic metabolites after ingestion of tropical highland blackberry (Rubus adenotrichus) juice. Food Research International, 55, 161-169.

11. García-Villalba, R., Espín, J.C., Tomás-Barberán, F.A. (2016). Chromatographic and spectroscopic characterization of urolithins for their determination in biological samples after the intake of foods containing ellagitannins and ellagic acid. Journal of Chromatography A, 1428, 162-175.

12. Giampieri, F., Tulipani, S., Alvarez-Suarez, J.M., Quiles, J.L., Mezzetti, B., Battino, M. (2012). The strawberry: composition, nutritional quality and impact on human health. Nutrition, 28(1), 9-19.

13. González-Barrio, R., Truchado, P., Ito, H., Espín, J.C., Tomás-Barberan, F.A. (2011). UV and MS identification of urolithins and nasutins, the bioavailable metabolites of ellagitannins and ellagic acid in different mammals. Journal of Agricultural and Food Chemistry, 59(4), 1152-1162.

14. Horwitz, W., Latimer, G.W. (2007). Official Methods of Analysis of AOAC International, 2005, 18th edn. AOAC International, USA.

15. Ito, H. (2011). Metabolites of the ellagitannin geraniin and their antioxidant activities. Planta Medica, 77(1), 1110-1115.

16. Ito, H., Iguchi, A., Hatano, T. (2008). Identification of urinary and intestinal bacterial metabolites of ellagitannin geraniin in rats. Journal of Agricultural and Food Chemistry, 56(2), 393-400.

17. Jurgoński, A., Juśkiewicz, J., Fotschki, B., Kołodziejczyk, K., Milala, J., Kosmala, M., Grzelak-Błaszczyk, K., Markiewicz, L. (2017). Metabolism of strawberry mono- and dimeric ellagitannins in rats fed a diet containing fructo-oligosaccharides. European Journal of Nutrition, 56(2), 853-864.

18. Juśkiewicz, J., Król, B., Kosmala, M., Milala, J., Zduńczyk, Z. (2015). Physiological properties of ellagitannin-rich preparations obtained from strawberry pomace using different extraction methods. Polish Journal of Food and Nutrition Sciences, 65(3), 199-209.

19. Karlińska, E., Pecio, Ł., Macierzyński, J., Stochmal, A., Kosmala, M. (2019). Structural elucidation of the ellagitannin with a molecular weight of 2038 isolated from strawberry fruit (Fragaria ananassa Duch.) and named fragariin A. Food Chemistry, 296, 109-115.

20. Kawabata, K., Yoshioka, Y., Terao, J. (2019). Role of intestinal microbiota in the bioavailability and physiological functions of dietary polyphenols. Molecules, 24(2), art. no. 370.

21. Kennedy, J.A., Jones, G.P. (2001). Analysis of proanthocyanidins cleavage products following acid-catalysis in the presence of excess phloroglucinol. Journal of Agricultural and Food Chemistry, 49(4), 1740-1746.

22. Kosmala, M., Jurgoński, A., Juśkiewicz, J., Karlińska, E., Macierzyński, J., Rój, E., Zduńczyk, Z. (2017). Chemical composition of blackberry press cake, polyphenolic extract, and defatted seeds, and their effects on cecal fermentation, bacterial metabolites, and blood lipid profile in rats. Journal of Agricultural and Food Chemistry, 65(27), 5470-5479.

23. Kosmala, M., Zduńczyk, Z., Juśkiewicz, J., Jurgoński, A., Karlińska, E., Macierzyński, J., Janczak, R., Rój, E. (2015). Chemical composition of defatted strawberry and raspberry seeds and the effect of these dietary ingredients on polyphenol metabolites, intestinal function, and selected serum parameters in rats. Journal of Agricultural and Food Chemistry, 63(11), 2989-2996.

24. Landete, J.M. (2011). Ellagitannins, ellagic acid and their derived metabolites: a review about source, metabolism, functions and health. Food Research International, 44(5), 1150-1160.

25. Larrosa, M., González-Sarrías, A., García-Conesa, M.T., Tomás-Barberán, F.A., Espín, J.C. (2006). Urolithins, ellagic acid-derived metabolites produced by human colonic microflora, exhibit estrogenic and antiestrogenic activities. Journal of Agricultural and Food Chemistry, 54(5), 1611-1620.

26. Larrosa, M., González-Sarrías, A., Yanez-Gascon M.J., Selma M.V., Azorin-Ortuno M., Toti S., Tomás-Barberán, F.A., Dolara P., Espín, J.C. (2010). Anti-inflammatory properties of a pomegranate extract and its metabolite urolithin-A in a colitis rat model and the effect of colon inflammation on phenolic metabolism. Journal of Nutritional Biochemistry, 21(8), 717-725.

27. Milala, J., Kosmala, M., Karlińska, E., Juśkiewicz, J., Zduńczyk, Z., Fotschki, B. (2017). Ellagitannins from strawberries with different degree of polymerization showed different metabolism through gastrointestinal tract of rats. Journal of Agricultural and Food Chemistry, 65(49), 10738-10748.

28. Nowicka, A., Kucharska, A.Z., Sokół-Łętowska, A., Fecka, E. (2019). Comparison of polyphenol content and antioxidant capacity of strawberry fruit from 90 cultivars of Fragaria $\times$ ananassa Duch. Food Chemistry, 270, 32-46.

29. Okva, K., Tamoseviciute, E., Ciziute, A., Pokk, P., Ruksenas, O., Nevalainen, T. (2006). Refinements for intragastric gavage in rats. Scandinavian Journal of Laboratory Science, 33(4), 243-252.

30. Piwowarski, J.P., Granica, S., Stefańska, J., Kiss, A.K. (2016). Differences in metabolism of ellagitannins by human gut microbiota ex vivo cultures. Journal of Natural Products, 79(12), 3022-3030.

31. Reeves, P.G. (1997). Components of the AIN-93 diets as improvements in the AIN-76A diet. Journal of Nutrition, 127, 838-841.

32. Romo-Vaquero, M., Cortes-Martin, A., Loria-Kohen, V., Ramirez-de-Molina, A., Garcia-Mantrana, I., Collado, C.M., Espín, J.C., Selma, M.V. (2019). Deciphering the human gut microbiome of urolithin metabotypes: association with enterotypes and potential cardiometabolic health implications. Molecular $\mathrm{Nu}$ trition and Food Research, 63(4), art. no. 1800958.

33. Saha, P., Yeoh, B.S., Singh, R., Chandrasekar, B., Vemula, P.K., Haribabu, B., Vijay-Kumar, M., Jala, V.R. (2016). Gut microbiota conversion of dietary ellagic acid into bioactive phytoceutical urolithin A inhibits heme peroxidases. PLoS ONE, 11(6), art. no. e0156811.

34. Sandhu, A.K., Miller, M.G., Thangthaeng, N., Scott, T.M., Shukitt-Hale, B., Edirisinghe, I., Burton-Freeman, B. (2018). Metabolic fate of strawberry polyphenols after chronic intake in healthy older adults. Food \& Function, 9(1), 96-106.

35. Sójka, M., Klimczak, E., Macierzynski, J., Kołodziejczyk, K. (2013). Nutrient and polyphenolic composition of industrial strawberry press cake. European Food Research and Technology, 237(6), 995-1007.

36. Tomás-Barberán, F.A., Gonzalez-Sarrias, A., Garcia-Villalba, R., Nunez-Sanchez, M.A., Selma, M.V., Garcia-Conesa, M.T., Espín, J.C. (2017). Urolithins, the rescue of "old" metabolites to understand a "new" concept: Metabotypes as a nexus among phenolic metabolism, microbiota dysbiosis, and host health 
status. Molecular Nutrition and Food Research, 61(1), art no. 1500901.

37. Toney, A.M., Fan, R., Xian, Y., Chaidez, V., Ramer-Tair, A.E., Chung, S. (2019). Urolithin A, a gut metabolite, improves insulin sensitivity through augmentation of mitochondrial function and biogenesis. Obesity, 27(4), 612-620.

38. Van de Velde, F., Esposito, D., Grace, M.H., Pirovani, M.E., Lila, M.A. (2019). Anti-inflammatory and wound healing properties of polyphenolic extracts from strawberry and blackberry fruits. Food Research International, 121, 453-462.
39. Vrhovsek, U., Guella, G., Gasperotti, M., Pojer, E., Zancato, M., Mattivi F. (2012). Clarifying the identity of the main ellagitannin in the fruit of the strawberry, Fragaria vesca and Fragaria ananassa Duch. Journal of Agricultural and Food Chemistry, 60(10), 2507-2516.

40. Wang, J., Hihara, E. (2004). A unified formula for calculating body surface area of humans and animals. European Journal of Applied Physiology, 92(1-2), 13-17.

Submitted: 28 June 2019. Revised: 8 August 2019. Accepted: 21 August 2019. Published on-line: 1 October 2019. 